\title{
Editorial
}

Pensar en Movimiento:

Revista de Ciencias del Ejercicio y la Salud EISSN 1659-4436

Vol. 17, № 1, pp. 1- 3

Inicia $1^{\circ}$ de enero, cierre al 30 de junio de 2019

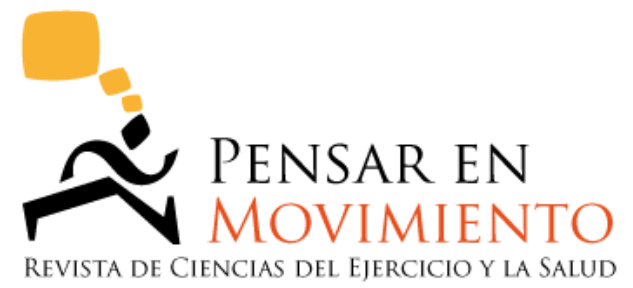

\section{LOS CAMBIOS RADICALES DE LA CIENCIA ABIERTA}

\author{
Luis Fernando Aragón-Vargas, Ph.D., FACSM \\ Centro de Investigación en Ciencias del Movimiento Humano \\ Universidad de Costa Rica, Costa Rica
}

Publicado: 2019-05-09

En Pensar en Movimiento nos debatimos constantemente entre las mejoras y el crecimiento, por un lado, y la necesidad de mantener los procesos suficientemente ágiles y sencillos para las personas que colaboran con nosotros como autoras o revisoras, por el otro. Nuestra línea editorial nos lleva a continuar adhiriéndonos a algunas tendencias de las publicaciones de punta en Acceso Abierto, como la revisión abierta por pares (optativa, ver Mora-Campos, A., 2015) y la publicación de las bases de datos crudos correspondientes a los manuscritos (altamente recomendada). Esto lo hacemos no solamente para facilitar el acceso libre a la información, sino para promover la ciencia transparente y reproducible.

Uno de los requisitos que vamos a comenzar a exigir es ligeramente engorroso, pero altamente valioso, aunque no se pueda decir que sea un cambio radical: el autor o la autora principal de cada manuscrito en nuestra revista deberá obtener un identificador único ORCID (ORCID ID) e incluirlo con su envío. Esto ha sido opcional en los metadatos sobre los autores, pero muy pronto va a ser obligatorio. La organización ORCID “...proporciona un identificador digital persistente que lo distingue a usted de todos los otros investigadores y, por medio de la integración en flujos de trabajo de investigación clave, como presentación de manuscritos y subvenciones, acepta enlaces automatizados entre usted y sus actividades profesionales, garantizando que su trabajo sea reconocido." (Disponible en https://orcid.org/). Entre las ventajas del ORCID ID están el ser gratuito, la utilización posterior tantas veces como se desee sin necesidad de volver a inscribirse, el permitir identificarse con gran facilidad en una variedad de plataformas informáticas, y la capacidad de reunir bajo una sola identidad-la suya-todas sus publicaciones y obras que probablemente aparecen bajo distintas variaciones de su nombre (con uno o dos apellidos, con caracteres especiales, con errores, etc.). Para más información, puede visitar https://orcid.org/about/what-is-orcid, disponible tanto en inglés como en español. Por sus ventajas, PENSAR EN MOVIMIENTO recomienda que todos los autores obtengan y reporten su ORCID al ingresar los metadatos del manuscrito. Entre las posibles desventajas está el hecho de que, al ser un registro que pertenece a un ente externo, algunas 
personas desconfían de que podría desaparecer o mudarse a un modelo comercial en el cual haya que pagar por su utilización. La forma de contrarrestar eso sería no depender únicamente del $O R C I D$ $I D$, sino crear un sistema propio de registro o utilizar varios a la vez.

Otro cambio importante tiene que ver con los preprints. Estos son manuscritos en versión preliminar, no revisada aún por pares, que se colocan en distintos tipos de repositorios. Bajo estas condiciones, no se consideran formalmente publicaciones, aunque tienen la ventaja de poner a disposición de la comunidad científica la información con más prontitud. Como desventajas, está el hecho de que la información divulgada aún no ha estado sujeta a la revisión por pares; además, que se podría ver afectado el proceso de revisión a ciegas por los pares académicos. Por esto último, muchas revistas que promueven el depósito de preprints someten los manuscritos a revisión abierta por pares. Conforme a las tendencias actuales, es sumamente deseable que las revistas científicas no solamente acepten para revisión manuscritos que podrían estar ya disponibles como preprint en un repositorio, como ha sido la política de PENSAR EN MOVIMIENTO desde hace muchos años, sino que además ofrezcan su propio repositorio en el cual los autores puedan hacer el depósito. Esta política ya la están utilizando muchas revistas de Acceso Abierto, y será muy pronto política oficial de nuestra revista. Estamos trabajando para ofrecer esta opción como institución Universidad de Costa Rica.

La ciencia mundial se dirige consistentemente hacia la transparencia de los datos. Esta transparencia significa que los datos utilizados para un artículo que se publica deben referenciarse claramente (si ya estuvieran disponibles) o, de otro modo, deberían publicarse o ponerse a disposición junto al manuscrito, en un formato accesible e inteligible; en cualquier caso, el manuscrito deberá citar debidamente la base de datos publicada. El objetivo de esto es que otros científicos puedan hacer sus propios cálculos o verificar los análisis estadísticos presentados por los autores (a eso se le llama promover la reproducibilidad de la ciencia). Esta práctica facilitará enormemente, entre otras cosas, la realización de metaanálisis, ya que ocasionalmente quien hace este tipo de revisión se ve en la obligación de contactar a los autores de uno o varios manuscritos en los cuales no se reportaron la media y desviación estándar de cada variable dependiente, tanto para el grupo control como para el grupo experimental. También obliga a los autores a ser más cuidadosos en sus análisis. Por otra parte, estas bases de datos están siendo citadas y cuentan también como parte de la producción científica de los investigadores. La transparencia de los datos es quizás uno de los aspectos más controversiales de la ciencia abierta, pues la costumbre de las prácticas anteriores nos hace temer que nos roben la información o que nos roben una metodología muy refinada. También hay recelo por la falta de legislación clara al respecto, así como por el posible mal uso de los datos por no comprenderlos bien. Por esto último, es sumamente importante curar y estandarizar los resultados antes de publicar una base de datos.

La transparencia también se refiere a que toda la buena ciencia se publique y esté disponible, no solamente aquellos estudios que arrojan resultados estadísticamente significativos. Para ello, PENSAR EN MOVIMIENTO facilitará la publicación de aquellos estudios cuya justificación y metodología sean sólidos, aún si los resultados son nulos o no muestran diferencias estadísticamente significativas entre grupos o tratamientos. Esto permite que el conocimiento sobre un fenómeno en particular sea más completo, evitando lo que se ha llamado el sesgo de los resultados positivos. 
Otros cambios están todavía pendientes, pero tenemos total apertura a la publicación de metodologías detalladas novedosas para la investigación de distintos temas, así como de programas o código de computación para la solución de distintos problemas o el cálculo de variables específicas. Estas publicaciones especiales, al igual que los archivos de datos crudos, se publicarán asociados a los manuscritos respectivos, pero serán citables de manera independiente y tendrán su propio DOI.

Finalmente, Pensar en Movimiento ha sido flexible, y lo seguirá siendo, con los límites establecidos en la extensión de los artículos (número de palabras) y en el número de referencias. Por esa razón, hemos ajustado nuestras instrucciones a los autores para establecer guías generales, no límites.

Con estos ajustes, seguimos avanzando hacia la calidad científica, además de apegarnos a las tendencias más importantes de la divulgación de la ciencia.

\section{Referencias}

Mora-Campos, A. (2015). Nuevas formas de revisión por pares en revistas científicas: revisión abierta / open review. Pensar En Movimiento: Revista De Ciencias Del Ejercicio Y La Salud, 13(1), 1-4. https://doi.org/10.15517/pensarmov.v13i1.19942

(2012) DORA: Declaración De San Francisco Sobre La Evaluación De La Investigación. Descargado el 2019/03/15 de https://sfdora.org/read/es/

Nosek, B. A., et al. (2015). Promoting an open research culture. Science 348(6242):1422-1425. DOI:

10.1126/science.aab2374. Descargado

2019/03/15

de http://science.sciencemag.org/content/348/6242/1422.full

Luis Fernando Aragón Vargas, Ph.D., FACSM

Director, PENSAR EN MOVIMIENTO

Universidad de Costa Rica 